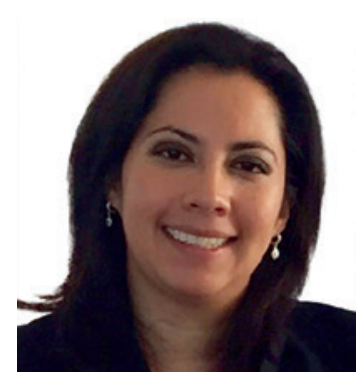

\title{
FPI: Mejorar la sobrevida mediante la evaluación y el tratamiento de los trastornos respiratorios del dormir
}

Zaira Romero-López

Instituto Mexicano del Seguro Social (IMSS), UMAE Hospital General Centro Médico Nacional La Raza, Ciudad de México, México

Resumen de Vozoris NT, Wilton AS, Austin PC, et al.: Morbidity and mortality reduction associated with polysomnography testing in idiopathic pulmonary fibrosis: a population-based cohort study. BMC Pulm Med. 2021;21(1):185.

\section{Keywords}

Sleep testing - Sleep breathing disorder - Pulmonary fibrosis . Health outcomes research . Health administrative database research

\begin{abstract}
Background: It is not well-known if diagnosing and treating sleep breathing disorders among individuals with idiopathic pulmonary fibrosis (IPF) improves health outcomes. We evaluated the association between receipt of laboratory-based polysomnography (which is the first step in the diagnosis and treatment of sleep breathing disorders in Ontario, Canada) and respiratoryrelated hospitalization and all-cause mortality among individuals with IPF.
\end{abstract}

Methods: We used a retrospective, population-based, cohort study design, analyzing health administrative data from Ontario, Canada, from 2007 to 2019. Individuals with IPF were identified using an algorithm based on health administrative codes previously developed by IPF experts. Propensity score matching was used to account for potential differences in 41 relevant covariates between individuals that underwent polysomnography (ex- posed) and individuals that did not undergo polysomnography (controls), in order minimize potential confounding. Respiratoryrelated hospitalization and all-cause mortality were evaluated up to 12 months after the index date.

Results: Out of 5044 individuals with IPF identified, 201 (4.0\%) received polysomnography, and 189 (94.0\%) were matched to an equal number of controls. Compared to controls, exposed individuals had significantly reduced rates of respiratory-related hospitalization (hazard ratio [HR] 0.43, 95\% confidence interval [Cl] $0.24-0.75), p=0.003)$ and all-cause mortality (HR 0.49, 95\% Cl $0.30-0.80), p=0.004)$. Significantly reduced rate of respiratoryrelated hospitalization (but not all-cause mortality) was also observed among those with $>=1$ respiratory-related hospitalization ( $\mathrm{HR} 0.38,95 \% \mathrm{Cl} 0.15-0.99)$ and systemic corticosteroid receipt (HR $0.37,95 \% \mathrm{Cl} 0.19-0.94)$ in the year prior to the index date, which reflect sicker subgroups of persons.

Conclusions: Undergoing polysomnography was associated with significantly improved clinically-important health outcomes among individuals with IPF, highlighting the potential importance of incorporating this testing in IPF disease management.

(c) 2021 Vozoris, Wilton, Austin, Kendzerska, Ryan, Gershon 


\section{Transferencia en la práctica}

\section{Contexto del estudio}

Las enfermedades pulmonares intersticiales difusas (EPID), son un grupo de enfermedades pulmonares crónicas progresivas con alta morbi-mortalidad. Las guías internacionales en relación con las EPID hacen énfasis en las distintas comorbilidades que pueden acompañar a este tipo de enfermedades incluyendo: enfermedad por reflujo gastroesofágico, la hipertensión pulmonar y la apnea obstructiva del sueño. Estudios previos han determinado una prevalencia del $17-88 \%$ de la apnea obstructiva del sueño (AOS), en pacientes con diagnóstico de EPID, en comparación con un 2-4\% en pacientes adultos sanos [1]. La fibrosis pulmonar idiopática (FPI), es el tipo más común de EPID con una mediana de supervivencia de 3-4 años, se estima que los trastornos respiratorios del dormir son frecuentes en esta entidad y que pueden empeorar dicha enfermedad mediante diferentes mecanismos, incluyendo el mecanismo de hipoxemia intermitente y re-oxigenación valorada en modelos animales, la cual promueve inflamación mediante vías oxidativas, así como el incremento en los niveles de IL-6 reflejando incremento de la permeabilidad de la pared alveolar y promoviendo lesión alveolar. Algunos estudios sugieren que los volúmenes pulmonares disminuidos en la EPID podrían promover la AOS al reducir la tracción en la tráquea y aumentar la colapsabilidad faríngea, otro mecanismo incluye la presencia de inspiraciones forzadas repetidas contra glotis cerrada, que se producen en la apnea obstructiva del sueño (AOS), pueden causar lesión por tracción recurrente del tejido pulmonar periférico y a su vez promover fibrosis pulmonar; sin olvidar que la AOS no tratada puede conducir a largo plazo al desarrollo de hipertensión pulmonar, que a su vez incrementa la mortalidad en pacientes con FPI [2]. El tratamiento con auxiliar de la ventilación con presión positiva en la vía aérea (PAP) y un adecuado apego estiman una mejor sobrevida en pacientes con FPI y AOS, sin haber mejoría en las exacerbaciones de FPI por otras causas que requirieron hospitalización.

El objetivo del estudio de Nicholas T. Vozoris y colegas, comentado aquí, fue evaluar la asociación entre la realización de polisomnografía (PSG) considerada estándar de oro para el diagnóstico de AOS, la hospitalización y la mortalidad por causas respiratorias en pacientes con diagnóstico de FPI. La hipótesis de los autores fue que los individuos que se someten a una PSG tendrán una menor tasa de hospitalización y mortalidad relacionadas con las vías respiratorias, que aquellos que no se someten a tales pruebas.

\section{Resultados del estudio}

El estudio de Vozoris et al. es un estudio de cohorte retrospectiva, incluyendo un grupo control. La población de estudio fueron los residentes de Ontario con diagnóstico de FPI de 66 años o más, identificados mediante un algoritmo desarrollado por un grupo de expertos en FPI reconocidos a nivel internacional, mediante criterios clínicos, de gabinete o definitivo (biopsia), el resultado final primario fue la hospitalización relacionada con el sistema respiratorio (insuficiencia respiratoria, neumonía, hipertensión pulmonar). Y el resultado secundario fue la mortalidad por todas las causas. Lo individuos se evaluaron con una máxima de seguimiento de doce meses después o hasta la fecha de la muerte o realización de trasplante pulmonar. El emparejamiento por puntuación de propensión se utilizó para crear muestras emparejadas de individuos expuestos y de control en las características sociodemográficas y de salud de referencia para reducir el sesgo. Se seleccionó una proporción de coincidencia de 1:1, se desarrolló una puntuación de propensión para la recepción de PSG utilizando un modelo de regresión logística que incorpora 41 variables, incluidos múltiples marcadores de gravedad de la FPI (incluyendo antecedentes de exacerbación respiratoria, complicaciones de la insuficiencia cardiaca congestiva tratamiento sistémico con corticosteroides). Se seleccionó la espirometría como prueba para la designación del grupo de control.

Se identificaron 5044 personas con FPI, de 66 años o más, de las cuales 201 (4,0\%) recibieron PSG durante el período de estudio. 189 $(94,0 \%)$ individuos expuestos se emparejaron con un número igual de controles. Se realizó un emparejamiento de puntajes de propensión, equilibrando 41 covariables, incluidos múltiples marcadores de gravedad de la FPI, estado general de salud, comorbilidades y utilización del sistema de atención médica.

En la cohorte emparejada por puntuación de propensión, en comparación con los controles, los individuos sometidos a PSG tuvieron tasas significativamente reducidas de hospitalización relacionada con las vías respiratorias (HR 0,43, IC del 95\%: 0,24-0,75, $p=0,003$, NNT 11) y morbilidad por todas las causas.

El lugar de realización del estudio Ontario, Canadá, los trastornos respiratorios del dormir se diagnostican únicamente mediante PSG, y las pruebas son obligatorias antes de iniciar los tratamientos adecuados.

Se consideró la población mayor a 66 años representativa en este estudio, debido a que el $70 \%$ o más de las personas afectadas tienen más de 65 años, se excluyeron a pacientes que recibieron cuidados paliativos, así como aquellos con antecedentes de realización de PSG previa o uso de dispositivo de ventilación u oxígeno suplementario.

La incidencia acumulada de hospitalización relacionada con las vías respiratorias fue significativamente menor y la probabilidad de supervivencia fue significativamente mayor entre los individuos sometidos a PSG frente a los controles. En similitud con los hallazgos reportados por Kolilekas et al. [3], que reporta en un estudio prospectivo, que la categoría más baja de saturación por pulsioximetría de oxígeno $\left(\mathrm{SpO}_{2}\right)$ se correlaciona directamente con la supervivencia, lo que implica que la AOS y la hipoxemia se correlacionan con la mortalidad relacionada con la enfermedad en pacientes con EPID.

\section{Conclusiones y recomendaciones para la práctica}

Este estudio comentado demuestra el hallazgo de que someterse a PSG se asocia con tasas significativamente más bajas de hospita- 
lización relacionada con las vías respiratorias y mortalidad por todas las causas entre las personas con FPI, en la población estudiada. Así también, los eventos posteriores a la PSG con relación al diagnóstico y el tratamiento de los trastornos respiratorios del dormir explican los beneficios observados, y la PSG refleja un marcador para estos eventos, sin embargo, no todos los marcadores de gravedad, ni el algoritmo fueron validados.

La realización de una PSG no significa que el trastorno respiratorio del dormir se haya establecido en un individuo o que a pesar de haber sido diagnosticado haya sido tratado de forma adecuada.

El paciente con diagnóstico de EPID cuenta con más comorbilidades dentro de su fisionomía, que tiene efecto en la sobrevida, los trastornos del dormir no se sospechan de primera intención en el abordaje de estos pacientes, por lo que llevaría a complicaciones tempranas como hipertensión pulmonar y cor pulmonale [4].

Este estudio nos brinda un panorama en relación a que el estudio de PSG se realiza con poca frecuencia en la población con diagnóstico de FPI, una enfermedad que es progresiva y con opciones limitadas de tratamiento.

Los resultados plantean la posibilidad de que la evaluación y el tratamiento adecuado de los trastornos respiratorios del dormir pueden brindar beneficios a la enfermedad en cuanto a sobrevida.
Se requieren estudios de seguimiento a largo plazo para evaluar el papel del diagnóstico y tratamiento oportuno de los trastornos del dormir en la sobrevida de estos pacientes y si además hay algún beneficio en mejorar su calidad de vida.

\section{Disclosure Statement}

Por la presente la autora declara que no tiene conflictos de interés con respecto a esta transferencia de conocimientos.

\section{Referencias}

1 Lee JH, Park CS, Song JW: Obstructive sleep apnea in patients with interstitia lung disease: Prevalence and predictive factors. PLoS One. 2020;15(10): e0239963.

2 Schiza SE, Bouloukaki I, Bolaki M, et al.: Obstructive sleep apnea in pulmonary fibrosis. Curr Opin Pulm Med. 2020;26(5):443-448.

3 Kolilekas L, Manali E, Vlami KA, et al.: Sleep oxygen desaturation predicts survival in idiopathic pulmonary fibrosis. J Clin Sleep Med. 2013;9(6):593601.

4 Reid T, Vennelle M, McKinley M, et al.: Sleep-disordered breathing and idiopathic pulmonary fibrosis-is there an association? Sleep Breath. 2015;19(2): 719-721.

Correspondencia:

Dra.Zaira Romero-López, zaira_doctora@yahoo.com.mx 